\title{
Association of Cytoplasmic CXCR4 with Loss of Epithelial Marker and Activation of ERK1/2 and AKT Signaling Pathways in Non-Small Cell Lung Cancer
}

Nabil F. Saba ${ }^{1 *}$, Yuxiang Wang ${ }^{1,2^{*}}$ Hongpeng Fu ${ }^{3}$, Lydia Koenig ${ }^{1}$, Fadlo R. Khuri ${ }^{1}$, Dong M. Shin ${ }^{1}$, Zhuo (Georgia) Chen ${ }^{\# 1}$

${ }^{1}$ Department of Hematology and Medical Oncology, Winship Cancer Institute, Emory University School of Medicine, Atlanta, GA

${ }^{2}$ Department of Pathology, Peking University Health Science Center, Beijing, China

${ }^{3}$ China Health Development \& Research Centor, Ministry of Health, Beijing, China ${ }^{\#}$ Corresponding Author: Zhuo (Georgia) Chen

1365-C Clifton Road, Suite C3086

Emory Winship Cancer Institute

Atlanta, GA 30322

Phone: (404)-778-3977

E-mail: gzchen@emory.edu

*These two authors contributed equally to the manuscript. 


\section{Abstract}

Objectives: Compelling evidence demonstrates that CXC-chemokine receptor 4 (CXCR4) is involved in tumor invasion, angiogenesis, metastasis, and resistance to chemotherapy in addition to being one of the co-receptors for T-tropic HIV entry into T cells. However, it still remains controversial as to how to identify functionally activated CXCR4 in tumor biopsies, which would assist in determining which patients may benefit from potential CXCR4-targeted therapy.

Materials and Methods: Immunohistochemistry (IHC) staining on archival tissues of patients with non-small cell lung cancer (NSCLC) was used to detect a panel of biomarkers, including phospho-ERK1/2, phospho-AKT, and E-cadherin which are relevant to downstream signaling of CXCR4 and epithelial to mesenchymal transition (EMT). We also examined whether subcellular localization of CXCR4 could help define possible activation of CXCR4.

Results: A total of 94 primary tumor tissue samples from patients with NSCLC were included. Sixty-six patients had both cytomembranous and nuclear staining of CXCR4, 22 had solely nuclear staining, 5 had solely cytomembranous staining, and 1 had negative staining. Cytoplasmic location of CXCR4 with or without nuclear location was associated with loss of the epithelial marker E-cadherin $(p=0.0015)$ and activation of ERK1/2 $(p=0.0121)$ and AKT $(p=0.0024)$, suggesting Epithelial-mesenchymal transition (EMT) in these tumors; while tumors with only nuclear location of CXCR4 were more indolent and preserved an epithelial phenotype.

Conclusions: Our study suggests that different subcellular localization of CXCR4 may be 
associated with different activation states; cytoplasmic CXCR4 seems to correlate with biomarkers changes associated with EMT in NSCLC.

Key words: CXCR4, epithelial-mesenchymal transition, E-cadherin, AKT, ERK1/2, NSCLC 


\section{Introduction}

Lung cancer remains the cancer with the highest mortality in the United States in both men and women, with an estimated 158,080 deaths in $2016 .{ }^{1}$ Non-small cell lung cancer (NSCLC) now accounts for more than $85 \%$ of lung cancers in the Western world. ${ }^{2}$ Due to the often asymptomatic and occult growth of NSCLC, most patients still present with advanced unresectable disease. ${ }^{3}$ Though tangible progress has been achieved with the introduction of chemotherapy and novel targeted agents, with noted advances with respect to classification, staging, diagnosis, and treatment of this disease, the mortality from NSCLC remains high. ${ }^{1}$ The introduction of targeted therapy has opened a new era for lung cancer treatment. ${ }^{4,5}$ Epidermal growth factor receptor (EGFR)-targeted therapy is an example of such targeted approach and is beneficial for patients who harbor specific mutations in the EGFR tyrosine kinase domain. ${ }^{6}$ Immunotherapy have also resulted in improved outcomes compared to cytotoxic chemotherapy for metastatic disease. ${ }^{7}$ Some patients with NSCLC will not, however, derive significant benefit from these targeted approaches, emphasizing the need for seeking personalized therapy as an optimal approach for treating patients. A substantial number of patients with known EGFR tyrosine kinase domain mutations who respond initially to EGFR-targeted therapy will develop resistance to EGFR tyrosine kinase inhibitor (TKI). ${ }^{8}$ This stresses the need for understanding the mechanisms behind acquired resistance to the different targeted agents.

CXCR4 is a G-protein coupled chemokine receptor (GPCR), that was first discovered as a co-receptor for T-tropic human immunodeficiency virus (HIV) entry into CD4+ T lymphocytes in $1996 .{ }^{9} \quad$ Fetal defects in the cardiovascular, hematopoietic system and central 
nervous systems were found in CXCR4-knockout SCID mice, resembling the lethal phenotypes found in mice with knockout of SDF-1, the ligand for CXCR4. ${ }^{10}$ CXCR4 was also found to play a crucial role in organ-specific metastasis of breast cancer. ${ }^{11,12}$ CXCR4 has been found to be highly expressed in multiple cancers, including lung cancer. ${ }^{13,14}$ After binding with its ligand SDF-1, CXCR4 plays a role in cancer proliferation, survival, invasion, angiogenesis, and drug resistance by activating its downstream ERK1/2, PI3K/AKT signaling pathways. ${ }^{15,16}$ Recent research has better defined the active crosstalk between tumor cells and their microenvironment and its critical role in tumor maintenance and progression. ${ }^{17}, 18$ SDF-1/CXCR4 is one of the key players in this crosstalk. Tumor cells as well as their surrounding fibroblasts, endothelial cells and inflammatory cells express CXCR4; $;^{19,20}$ furthermore, mesenchymal stem cells in the bone marrow, some local stromal cells of certain organs and even some tumor cells can secrete SDF-1. ${ }^{21}$ This complicated network facilitates the interaction of tumor cells with their environment and accelerates proliferation and metastasis. ${ }^{10}$ CXCR4 may therefore serve as a target for cancer therapy, since inhibiting CXCR4 could affect the growth of tumor cells and the tumor micro-environment.

There is clear evidence from cell line and animal models that activation of CXCR4 can promote tumor proliferation and/or invasion; ${ }^{22,23}$ thus, inhibition of CXCR4 by antagonists or knockdown could reduce metastasis. ${ }^{24,25}$ There appear to be several distinct patterns of CXCR4 staining, including nuclear staining and cytoplasmic/cytomembranous staining patterns. $^{26,27}$ We were interested in determining which staining pattern represents the functional form of CXCR4 that correlates with a more aggressive tumor phenotype. The results from different research groups remain controversial regarding this subject. ${ }^{28-30}$ 
Epithelial-mesenchymal transition (EMT) is a transient and reversible process that describes the gradual loss of intercellular adhesion and polarity of epithelial cells, and their transition to an isolated, motile and fibroblast-like phenotype. This process was first discovered in embryogenesis, and then recognized as a potential mechanism of cancer progression. Accumulating evidence shows that tumor cells undergoing EMT, which is usually initiated by abnormally activated PI3K/AKT or ERK1/2 pathways, ${ }^{31,32}$ then become more invasive and more resistant to anoikis and chemotherapy. ${ }^{33,34}$ The loss of the intercellular adhesion molecule E-cadherin is well accepted as a hallmark of EMT. Therefore, we investigated a panel of EMT-related markers, including E-cadherin, phospho-ERK1/2, and phospho-AKT, in addition to CXCR4, to help define the functional state of CXCR4 and to elucidate the association between the subcellular localization of CXCR4 and its functional state in NSCLC samples. 


\section{Materials and Methods}

\section{Human Tissues and Cell Lines}

A total of 94 formalin-fixed and paraffin-embedded primary tumor samples from patients with NSCLC who underwent surgical resection at Emory University Hospital from 1998 to 2002 following the regulation of the Health Insurance Portability and Accountability Act (HIPAA) were included. Patient information was collected and is summarized in Table 1. Limited information on survival existed as a large proportion of patients were lost to follow up given their early stage disease and curative nature of their surgical resections. We attempted however to correlate our histologic findings with the available clinical information.

Human NSCLC cell lines (H292, H322, A549--adenocarcinoma, H157, H460, 801D--large cell carcinoma, and H1703--squamous cell carcinoma) were obtained from Drs. Shi-Yong Sun (Emory University, Atlanta USA) and Yinglin Lu (Institute of Basic Medical Sciences, Beijing China) ${ }^{35-37}$. The cells were cultured in RPMI 1640 medium supplemented with $10 \%$ fetal bovine serum.

\section{Immunohistochemistry (IHC) Staining and Scoring}

VECTOR kits--Deparaffinization, rehydration, and antigen retrieval were followed by incubation of primary antibody at $4^{\circ} \mathrm{C}$ overnight. The primary antibodies included mouse E-cadherin (BD Biosciences), mouse CXCR4 (R\&D Systems), rabbit phospho-AKT (Cell Signaling Tech), and rabbit phospho-ERK1/2 (Cell Signaling Tech). The secondary antibodies were incubated for $20 \mathrm{~min}$ at room temperature, followed by DAB staining and 
counterstaining with hematoxylin.

The intensity of IHC staining was measured using a numerical scale $(0=$ no expression, $1+=$ weak expression, $2+=$ moderate expression, and $3+=$ strong expression $)$ and assessed as Weight Index [WI $=\%$ Positive Stain $(>0)$ in Tumor $\times$ Intensity Score].

\section{Western Blot Analysis}

NSCLC cell lines were grown to 70-80\% confluence, serum starved for 24 hours, and treated with SDF-1. Cell lysates were collected for Western blot analysis with primary antibody: mouse CXCR4 (Abcam Inc.), rabbit GAPDH (Upstate Inc), rabbit histone H3 - a nuclear marker (Cell Signaling Tech), rabbit phopho-AKT (Cell Signaling Tech), rabbit phopho-ERK1/2 (Cell Signaling Tech), and the secondary antibodies.

\section{Statistical Analysis}

Pearson Chi-square test was used to correlate the different subcellular localization groups of CXCR4 with clinical data. ANOVA analysis with SPSS version 17 (SPSS, Chicago, IL, USA) was used to explore the correlation between CXCR4 location and the EMT markers. P-values less than 0.05 were considered statistically significant. 


\section{Results}

Expression of CXCR4 in Tumor Tissue and Normal Bronchial Epithelium

As illustrated in Table 1, of all 94 patients 46 patients had T1 disease, 43 had T2, 1 had T3, and 4 had T4. Seventy-three patients had N0 disease, 18 had N1, and 3 had N2. For histological type, 56 cases were adenocarcinoma, 34 were squamous cell carcinoma, and 4 were large cell carcinoma.

CXCR4 expression was detected in 98.9\% (93/94) of samples. Of interest were the 3 distinctive expression patterns for CXCR4 including cytoplasmic, nuclear, and cytoplasmic/nuclear expression (Figure 1). The normal bronchial epithelium was negatively stained by CXCR4 (data not shown).

Correlation of CXCR4 Localization with Loss of Epithelial Marker and Activation of phospho-ERK and phospho-AKT

To determine whether the different subcellular locations of CXCR4 are related to different activated states, we used a panel of EMT-related markers, including E-cadherin, phospho-ERK1/2 and phospho-AKT, to better define the activated state of CXCR4. The cytoplasmic localization of CXCR4 was correlated with loss of the epithelial marker E-cadherin and activation of phospho-ERK1/2 and phospho-AKT pathways. The expression levels of phospho-AKT and phospho-ERK1/2 were both significantly higher ( $\mathrm{p}=0.0024$ and $\mathrm{p}=0.0121$, respectively) in the CXCR4 cytoplasmic and cytoplasmic/nuclear localization groups than in the CXCR4 nuclear localization only group. The expression level of 
E-cadherin in the CXCR4 cytoplasmic and cytoplasmic/nuclear localization groups was significantly lower $(\mathrm{p}=0.0015$ ) compared to the expression in the CXCR4 nuclear staining group (Figure 2).

\section{Patient Characteristics and Clinical Data Analysis}

The characteristics of the NSCLC patients are shown in Table 1. Ninety three out of 94 patients with positive staining of CXCR4 were classified into 3 groups according to the subcellular localization of CXCR4. The variables thought to be related with prognosis in NSCLC, such as gender, age, smoking and drinking history, T-stage, N-stage, tumor grade, chemotherapy, radiotherapy and overall survival were included. None of the available clinical characteristics correlated with different CXCR4localization groups. Unfortunately, as a high proportion of patients sought additional follow up and therapy outside our institution and was therefore lost to follow up, it was not possible to correlate the tissue biomarker profiles with clinical outcome such as progression free survival or overall survival.

\section{Expression Pattern of CXCR4 in NSCLC Cell Lines}

In order to clarify whether CXCR4 is expressed in different histological types of NSCLC, different types of cell lines were used including squamous cell carcinoma-H1703, adenocarcinoma-H292, H322, A549, and large cell carcinoma-H157, H460. All cell lines expressed CXCR4 (Figure 3a), as shown by Western blot. To determine whether cell lines have similar distribution patterns of CXCR4 as NSCLC tissues, we performed Western blot by isolating cytoplasmic and nuclear fractions of H292 and H1703 cell lines and confirmed 
that CXCR4 is located in both the cytoplasm and nuclei (Figure 3b).

Activation of ERK1/2 and/or AKT Pathways by SDF-1 in NSCLC cell lines

It has long been recognized that abnormally activated AKT and/or ERK signaling pathways are involved in the process of EMT. Furthermore, CXCR4 activation of the AKT and/or ERK signaling pathway has been reported in several cancer cell lines. ${ }^{38,39}$ To determine whether CXCR4 could activate these two signaling pathways in NSCLC cell lines, we used SDF-1, the ligand of CXCR4, to activate the cells. Western blot analysis on three NSCLC cell lines showed that the phosphorylation levels of AKT and/or ERK were significantly increased by stimulation with SDF-1 (Figure 4). 


\section{Discussion}

CXCR4 expression is associated with a more aggressive tumor behavior including NSCLC. ${ }^{13,}$

${ }^{40}$ In our present study, a positive expression of CXCR4 was detected in 93 out of 94 NSCLC cases, including squamous cell carcinoma, adenocarcinoma and large cell carcinoma, while the normal bronchial epithelium cells lacked the same expression. This suggests that CXCR4 expression is associated with tumorigenesis or progression in different histologic subtypes of NSCLC. Though more than $90 \%$ of tumor samples express CXCR4, only those with cytoplasmic location were more likely to have activated AKT and ERK1/2 together with decreased expression of E-cadherin, suggesting that tumors with these biomarker changes are destined to undergo EMT with transformation to spindle cell histology. When CXCR4 was only located in nuclei, tumor cells retained their epithelial phenotype and were free from activation of these two signaling pathways. Together with our cell line data showing that activation of CXCR4 by SDF-1 is followed by phosphorylation of AKT and/or ERK signaling pathways, we suggest that cytoplasmic CXCR4 may promote EMT and tumor progression. Therefore, subcellular localization of CXCR4 in addition to intensity of staining may be an important factor to take into account when evaluating this pathway in NSCLC. This notion is supported by a recent meta-analysis of expression of CXCR4 in NSCLC. ${ }^{41}$ It revealed that CXCR4 overexpression in the cytoplasm predicted poor overall survival, but elevated CXCR4 expression in the nucleus was positively associated with disease free survival. Other similar examples of molecular pathways include the IkappaB family, which translocate to the nucleus to promote gene transcription when being activated during tumorigenesis, ${ }^{42}$ and $\beta$-catenin which helps keep the intercellular adhesion complex and 
polarity of the epithelium intact when located at the membrane, but promotes cell proliferation in the nucleus. ${ }^{43}$

Evidence suggests that a cytomembrane expression of CXCR4 is associated with a shorter disease-free survival of patients with adenocarcinoma of the lung. ${ }^{30}$ Studies suggest that SDF-1/CXCR4 induces EMT through activating the AKT signaling pathway in oral squamous cell carcinoma ${ }^{44}$ and glioblastoma cell lines. ${ }^{45}$ There are, however, so far no data clarifying the association between the functional location of CXCR4 and EMT on a tissue level. According to our knowledge, this is the first study to explore the functional localization of CXCR4 in NSCLC samples.

The limitation of this current study is the lack of clinical and survival data, as most of the patients were in $\mathrm{T} 1$ or $\mathrm{T} 2$ stage (89/94) and a large number of patients were lost to follow up. Even though some tumors expressed cytoplasmic CXCR4 at the time of inspection, nuclear-localized CXCR4 in other tumors may have translocated to the cytoplasm during their progression. Our evaluation is therefore limited by these possibilities. Our data is relevant in that it shows that the cytoplasmic localization of CXCR4 is correlated with the EMT state, a finding that deserves to be further evaluated in a prospectively collected dataset with available clinical information. As most patients had early stage disease, our findings indicate that the expression of functional CXCR4 and EMT can occur in early stage tumors, which warrants further evaluation in this setting.

\section{Conclusions}

In summary, our results describe the different expression patterns of CXCR4 in various NSCLC histologic types types and demonstrate that the localization of CXCR4 is correlated 
with loss of the EMT marker in this disease. Our findings provide useful information for further studying these effects and offer a guide for future research aiming at better understanding of the role of CXCR4 and its prognostic significance in NSCLC.

\section{Acknowledgments}

We thank Drs. Anthea Hammond for her critical reading and editing, and Anthony A. Gal for providing the tissue samples. This study was supported by grants from NIH R21 CA125062, DOD W81XWH-07-1-0306 Project 5 to ZGC, and GCC Distinguished Scholar Award to ZGC and DMS.

\section{References}

1. Siegel RL, Miller KD, Jemal A. Cancer statistics, 2016. CA Cancer J Clin 2016;66:7-30.

2. Yano T, Haro A, Shikada Y, Maruyama, R. Maehara, Y. Non-small cell lung cancer in never smokers as a representative 'nonsmoking- associated lung cancer': epidemiology and clinical features. Int J Clin Oncol 2011;16:187-93.

3. Ramnath N, Dilling TJ, Harris LJ, Kim AW, Michaud GC, Balekian AA, et al. Treatment of Stage III Non-small Cell Lung Cancer: Diagnosis and Management of Lung Cancer, 3rd ed: American College of Chest Physicians Evidence-Based Clinical Practice Guidelines. Chest 2013 143:E314S-40S.

4. Ramalingam SS, Owonikoko TK, Khuri FR. Lung cancer: New biological insights and recent therapeutic advances. CA Cancer J Clin 2011;61:91-112.

5. Kohno T, Yokota J. How many tumor suppressor genes are involved in human lung 
carcinogenesis? Carcinogenesis 1999;20:1403-10.

6. Mounawar M, Mukeria A, Le Calvez F, Huang RJ, Renard H, Cortot A, et al. Patterns of EGFR, HER2, TP53, and KRAS mutations of p14arf expression in non-small cell lung cancers in relation to smoking history. Cancer Res 2007;67:5667-72.

7. Brahmer J, Reckamp KL, Baas P, Crinò L, Eberhardt WE, Poddubskaya E, et al., Nivolumab versus Docetaxel in Advanced Squamous-Cell Non-Small-Cell Lung Cancer. N Engl J Med 2015;373:123-35.

8. Kim Y, Ko J, Cui Z, Abolhoda A, Ahn JS, Ou SH, et al. The EGFR T790M mutation in acquired resistance to an irreversible second-generation EGFR inhibitor. Mol Cancer Ther 2012;11:784-91.

9. Feng Y, Broder CC, Kennedy PE, Berger EA. HIV-1 entry Cofactor: Functional cDNA cloning of a seven-transmembrane, G protein-coupled receptor. Science 1996;272 (5263):872-7.

10. Lapidot T. Mechanism of human stem cell migration and repopulation of NOD/SCID and B2mnull NOD/SCID mice. The role of SDF-1/CXCR4 interactions. Ann N Y Acad Sci 2001;938:83-95.

11. Müller A, Homey B, Soto H, Ge H, Catron D, Buchanan ME, et al. Involvement of chemokine receptors in breast cancer metastasis. Nature 2001;410:50-6.

12. Boimel PJ, Smirnova T, Zhou ZN, Wyckoff J, Park H, Coniglio SJ, et al. Contribution of CXCL12 secretion to invasion of breast cancer cells. Breast Cancer Res 2012;14:R23.

13. Wald O, Shapira OM, Izhar U. CXCR4/CXCL12 axis in non-small cell lung cancer (NSCLC) pathologic roles and therapeutic potential. Theranostics 2013;3:26-33. 
14. Al Zobair AA, Al Obeidy BF, Yang L, Yang C, Hui Y, Yu H, et al. Concomitant overexpression of EGFR and CXCR4 is associated with worse prognosis in a new molecular subtype of non-small cell lung cancer. Oncol Rep 2013;29:1524-32.

15. Ping YF, Yao XH, Jiang JY, Zhap LT, Yu SC, Jiang T, et al. The chemokine CXCL12 and its receptor CXCR4 promote glioma stem cell-mediated VEGF production and tumour angiogenesis via PI3K/AKT signalling. J Pathol 2011;244:344-54.

16. Liang Z, Brooks J, Willard M, Liang K, Yoon Y, Kang S, et al. CXCR4/CXCL12 axis promotes VEGF-mediated tumor angiogenesis through Akt signaling pathway. Biochem Biophys Res Commun 2007;359:716-22.

17. Yan $\mathrm{L}, \mathrm{Cai} \mathrm{Q}, \mathrm{Xu} \mathrm{Y}$. The ubiquitin-CXCR4 axis plays an important role in acute lung-infection-enhanced lung tumor metastasis. Clin Cancer Res 2013;19:4706-16. .

18. Zhang L, Ye SB, Ma G, Tang XF, Chen SP, He J, et al. The expressions of MIF and CXCR4 protein in tumor microenvironment are adverse prognostic factors in patients with esophageal squamous cell carcinoma. J Transl Med 2013; 11:60. doi: 10.1186/1479-5876-11-60.

19. Hosokawa Y, Hosokawa I, Ozaki K, Nakae H, Murakami K, Miyake Y, et al. CXCL12 and CXCR4 expression by human gingival fibroblasts in periodontal disease. Clin Exp Immunol 2005;141:467-74.

20. Bachelder RE, Wendt MA, Mercurio AM. Vascular endothelial growth factor promotes breast carcinoma invasion in an autocrine manner by regulating the chemokine receptor CXCR4. Cancer Res 2002;62:7203-6.

21. Yang D, Sun S, Wang Z, Zhu P, Yang Z, Zhang B. Stromal Cell-Derived Factor-1 
Receptor CXCR4-Overexpressing Bone Marrow Mesenchymal Stem Cells Accelerate Wound Healing by Migrating into Skin Injury Areas. Cell Reprogram 2013;15:206-15.

22. Arora S, Bhardwaj A, Singh S, Srivastava SK, McClellan S, Nirodi CS, et al. An undesired effect of chemotherapy: gemcitabine promotes pancreatic cancer cell invasiveness through ROS-dependent, NF- $\mathrm{BB}$ - and HIF-1 $\alpha$-mediated upregulation of CXCR4. J Biol Chem 2013; 288:21197-207.

23. Hong JS, Pai HK, Hong KO, Kim MA, Kim JH, Lee JI, et al CXCR-4 knockdown by small interfering RNA inhibits cell proliferation and invasion of oral squamous cell carcinoma cells. J Oral Pathol Med 2009;38:214-9.

24. Wang J, Cai J, Han F, Yang C, Tong Q, Cao T, et al. Silencing of CXCR4 blocks progression of ovarian cancer and depresses canonical Wnt signaling pathway. Int $J$ Gynecol Cancer 2011;21:981-7.

25. Huang EH, Singh B, Cristofanilli M, Gelovani J, Wei C, Vincent L, et al. A CXCR4 antagonist CTCE-9908 inhibits primary tumor growth and metastasis of breast cancer. $J$ Surg Res 2009;155:231-6.

26. Kato M, Kitayama J, Kazama S, Nagawa H. Expression pattern of CXC chemokine receptor-4 is correlated with lymph node metastasis in human invasive ductal carcinoma. Breast Cancer Res 2003;5:R144-50.

27. Wang L, Wang Z, Yang B, Yang Q, Wang L, Sun Y. CXCR4 nuclear localization follows binding of its ligand SDF-1 and occurs in metastatic but not primary renal cell carcinoma. Oncol Rep 2009;22:1333-9.

28. Spano JP, Andre F, Morat L, Sabatier L, Besse B, Combadiere C, et al. Chemokine 
receptor CXCR4 and early-stage non-small cell lung cancer: pattern of expression and correlation with outcome. Ann Oncol 2004; 15:613-7.

29. Na IK, Scheibenbogen C, Adam C, Stroux A, Ghdjar P, Thiel E, et al. Nuclear expression of CXCR4 in tumor cells of non-small cell lung cancer is correlated with lymph node metastasis. Hum Pathol 2008; 39 :1751-5.

30. Wagner PL, Hyjek E, Vazquez MF, Meherally D, Liu YF, Chadwick PA, et al. CXCL12 and CXCR4 in adenocarcinoma of the lung: association with metastasis and survival. $J$ Thorac Cardiovasc Surg 2009; 137:615-21.

31. Bakin AV, Tomlinson AK, Bhowmick NA, Moses HL, Arteaga CL. Phosphatidylinositol 3-kinase function is required for transforming growth factor beta-mediated epithelial to mesenchymal transition and cell migration. J Biol Chem 2000 ;275:36803-10.

32. Janda E, Lehmann K, Killisch I, Jechinger M, Herzig M, Downward J, et al. Ras and TGF[beta] cooperatively regulate epithelial cell plasticity and metastasis: dissection of Ras signaling pathways. J Cell Biol 2002;156:299-313.

33. Zuo JH, Zhu W, Li MY, Li XH, Yi H, Zeng GQ, et al. Activation of EGFR promotes squamous carcinoma SCC10A cell migration and invasion via inducing EMT-like phenotype change and MMP-9-mediated degradation of E-cadherin. J Cell Biochem 2011;112:2508-17.

34. Voulgari A, Pintzas A. Epithelial-mesenchymal transition in cancer metastasis: mechanisms, markers and strategies to overcome drug resistance in the clinic. Biochim Biophys Acta 2009;1796:75-90.

35. Zhang JQ, Wang Y, Wang T, Du ZY, Xu YJ, Lu YL. Differentially expressed gene in 
human giant-cell lung cancer lines with different metastatic potentials. Zhonghua Zhong Liu Za Zhi 2004;26:590-3.

36. Li, Y, Fan S, Koo J, Yue P, Chen ZG, Owonikoko TK, Ramalingam SS, Khuri FR, Sun S-Y. Eleveated expression of eukaryotic translation initiation factor $4 \mathrm{E}$ is associated with proliferation, invasion and acquired resistance to erlotinib in lung cancer. Cancer Biol \& Ther 2012;13:272-80.

37. Oh Y-T, Yue P, Wang D, Tong J-S, Chen ZG, Khuri FR, Sun S-Y, Suppression of death receptor 5 enhances cancer cell invasion and metastasis through activation of caspase-8/TRAF2-mediated signaling. Oncotarget 2015;6:41324-38.

38. Shen X, Artinyan A, Jackson D, Thomas RM, Lowy AM, Kim J. Chemokine receptor CXCR4 enhances proliferation in pancreatic cancer cells through AKT and ERK dependent pathways. Pancreas 2010;39(1):81-7.

39. Wu M, Chen Q, Li D, Li X, Li X, Huang C, et al. LRRC4 inhibits human glioblastoma cells proliferation, invasion, and proMMP-2 activation by reducing SDF-1 alpha/CXCR4-mediated ERK1/2 and Akt signaling pathways. J Cell Biochem 2008;103:245-55.

40. Gangadhar T, Nandi S, Salgia R. The role of chemokine receptor CXCR4 in lung cancer. Cancer Biol Ther, 2010;9:409-16.

41. Liu K, Bao C, Yao N, Miao C. Varlotto J, Sun Q et al., Expression of CXCR4 and non-small cell lung cancer prognosis: a meta-analysis. Int $J$ Clin Exp Med 2015;8:7435-45.

42. Lentsch $\mathrm{AB}$, Ward PA. Activation and regulation of NFkappaB during acute 
inflammation. Clin Chem Lab Med 1999;37:205-8.

43. Huber O, Korn R, McLaughlin J, Ohsugi M, Herrmann BG, Kemler R. Nuclear localization of beta-catenin by interaction with transcription factor LEF-1. Mech Dev 1996;59:3-10.

44. Onoue T, Uchida D, Begum NM, Tomizuka Y, Yoshida H, Sato M. Epithelial-mesenchymal transition induced by the stromal cell-derived factor-1/CXCR4 system in oral squamous cell carcinoma cells. Int J Oncol 2006;29:1133-8.

45. Lv B, Yang X, Lv S, Wang L, Fan K, Shi R, CXCR4 signaling induced epithelial-mesenchymal transition by PI3K/AKT and ERK Pathways in glioblastoma. Mol Neurobil 2015; 52:1263-8. 


\section{Figure Legends}

Fig. 1 Different staining patterns of CXCR4 in human NSCLC specimens. $A$. Nuclear staining pattern, the positive signal only located in nuclei $(n=22)$. B. Cytoplasmic staining pattern, the positive signal only located in cytoplasm $(n=5) . C$. Cytoplasmic+nuclear staining pattern, the positive signal distributed both in nuclei and cytoplasm $(\mathrm{n}=66) . D$. Negative staining pattern $(n=1)$.

Fig. 2 Localization of CXCR4 is correlated with loss of the EMT marker and activation of downstream signaling molecules. (a) Two representative cases of squamous cell carcinoma and adenocarcinoma are presented. $A, B, C$, and $D$ were from the same patient sample, in which CXCR4 was located in both cytoplasm and nuclei. Both AKT and ERK1/2 pathways were activated in this patient, while the expression of epithelial marker E-cadherin was dramatically decreased and distributed in a diffuse pattern rather than a normal membrane staining pattern. $E, F, G$, and $H$ were from another patient sample, in which CXCR4 was located only in nuclei. No activation of AKT and ERK1/2 was detected, while E-cadherin was expressed at a high level and well distributed in a linear pattern. These staining patterns are not histologically specific. (b) Expression levels of p-AKT, p-ERK and E-cadherin are correlated with the localization of CXCR4. The correlation of different locations of CXCR4 with p-AKT, p-ERK, and E-cadherin was evaluated statistically by ANOVA. The result shows that the expression levels of p-AKT and p-ERK are both significantly higher $(\mathrm{p}=0.0024$ and $\mathrm{p}=0.0121$, respectively) in samples with cytoplasmic and/or cytoplasmic/nuclear CXCR4 localization than in those with nuclear CXCR4 
localization. The expression level of E-cadherin in the CXCR4 cytoplasmic and cytoplasmic/nuclear localization groups was also significantly lower $(\mathrm{p}=0.0015)$ than that in the CXCR4 nuclear staining group (dashed line--mean value, solid line--median value).

Fig. 3 Western blot analysis of NSCLC cell lines is consistent with tissue IHC studies.

(a) Expression of CXCR4 in NSCLC cell lines. Western blot shows CXCR4 expression in different histological types (H1703--squamous cell carcinoma, H292, H322, A549-adenocarcinoma and H157, H460--large cell carcinoma) of human NSCLC cell lines. (b) Localization of CXCR4 in NSCLC cell lines. By extracting the cytoplasmic and nuclear fractions separately, CXCR4 could be detected in both cytoplasmic and nuclear compartments (histone H3 was used as a nuclear marker; GAPDH was used as cytoplasmic marker).

Fig. 4 The ligand of CXCR4, SDF-1, could activate ERK1/2 and/or AKT pathways in NSCLC cell lines. Western blot shows $100 \mathrm{ng} / \mathrm{ml}$ SDF- 1 could activate the ERK1/2 and/or AKT pathways in different types of NSCLC cell lines. 
Table 1. in this study

Patient characteristics. Baseline characteristics of the 93 patients that were included

\begin{tabular}{|c|c|c|c|c|c|}
\hline \multirow[t]{2}{*}{ Varieble } & \multirow[t]{2}{*}{ Categories } & \multicolumn{3}{|c|}{ Localization of CXCR4 } & \multirow{2}{*}{$\frac{\text { Pearson Chi-square }}{p \text {-value }}$} \\
\hline & & Nuclear & Nuclear+cytoplasmic & Cytoplasmic & \\
\hline \multirow[t]{2}{*}{ Gender } & Male & 11 & 34 & 3 & 0.883 \\
\hline & Female & 11 & 32 & 2 & \\
\hline Age & median & 63.41 & 67.17 & 69.8 & 0.167 \\
\hline \multirow[t]{2}{*}{ Smoking } & No & 8 & 18 & 1 & 0.647 \\
\hline & Yes & 14 & 48 & 4 & \\
\hline \multirow[t]{2}{*}{ Alcohol } & No & 19 & 58 & 5 & 0.689 \\
\hline & Yes & 3 & 8 & 0 & \\
\hline \multirow[t]{4}{*}{$\mathrm{T}-$ Class } & $\mathrm{T} 1$ & 10 & 31 & 4 & 0.317 \\
\hline & $\mathrm{T} 2$ & 9 & 33 & 1 & \\
\hline & T3 & 1 & 0 & 0 & \\
\hline & $\mathrm{T} 4$ & 2 & 2 & 0 & \\
\hline \multirow{3}{*}{$\mathrm{N}-$ Class } & No & 19 & 49 & 4 & 0.701 \\
\hline & N1 & 2 & 15 & 1 & \\
\hline & N2 & 1 & 2 & 0 & \\
\hline \multirow[t]{4}{*}{ Grade } & G1 & 3 & 6 & 0 & 0.468 \\
\hline & G2 & 16 & 42 & 2 & \\
\hline & G3 & 3 & 17 & 3 & \\
\hline & $\mathrm{N} / \mathrm{A}$ & 0 & 1 & 0 & \\
\hline \multirow[t]{2}{*}{ Chemotherapy } & No & 20 & 62 & 5 & 0.735 \\
\hline & Yes & 2 & 4 & 0 & \\
\hline \multirow[t]{2}{*}{ Radiotherapy } & No & 21 & 64 & 5 & 0.862 \\
\hline & Yes & 1 & 2 & 0 & \\
\hline OS (month) & mean & 58.82 & 63.11 & 64.2 & 0.898 \\
\hline
\end{tabular}




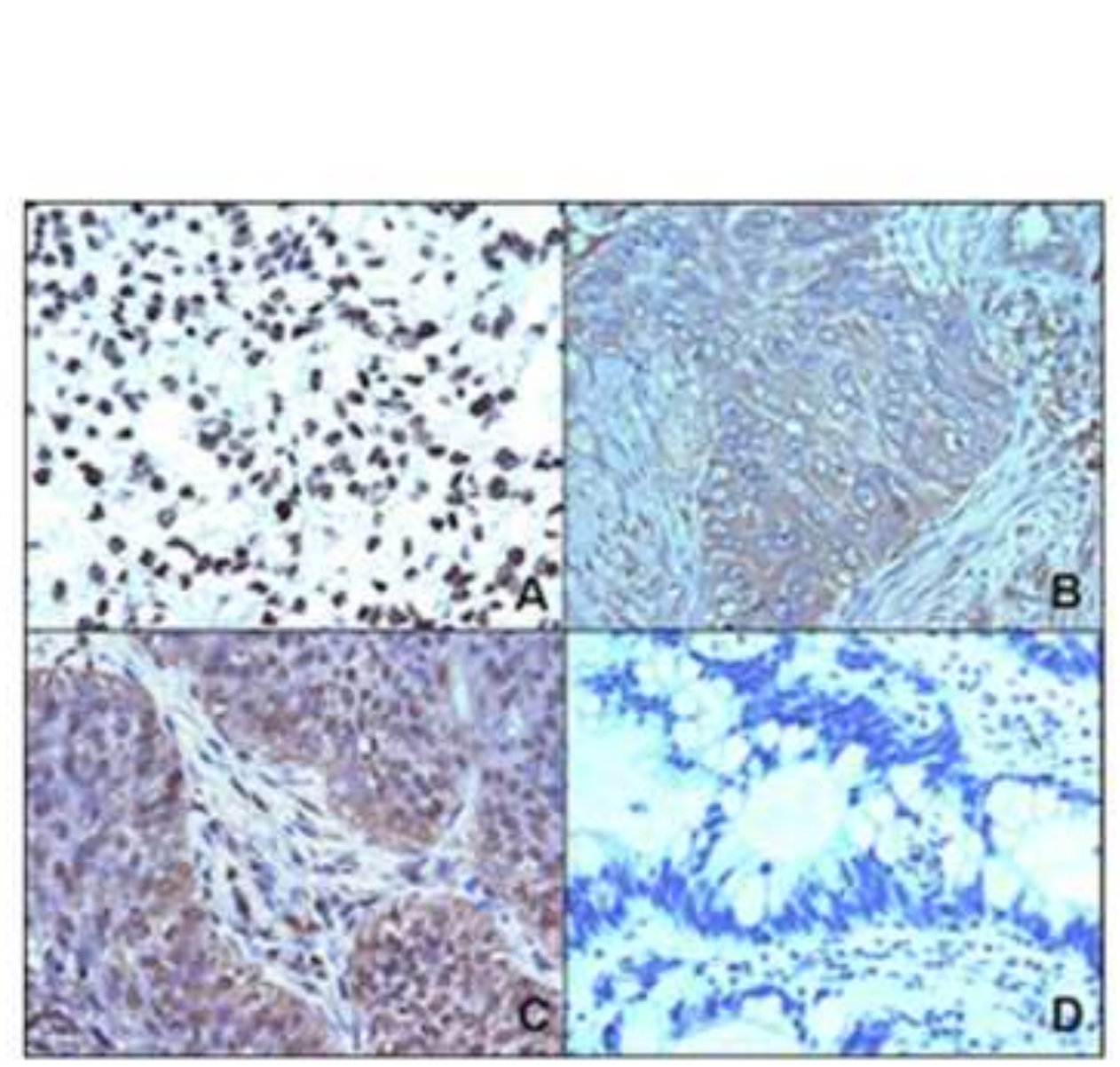

\section{Figure 1}




\section{Figure 2a}

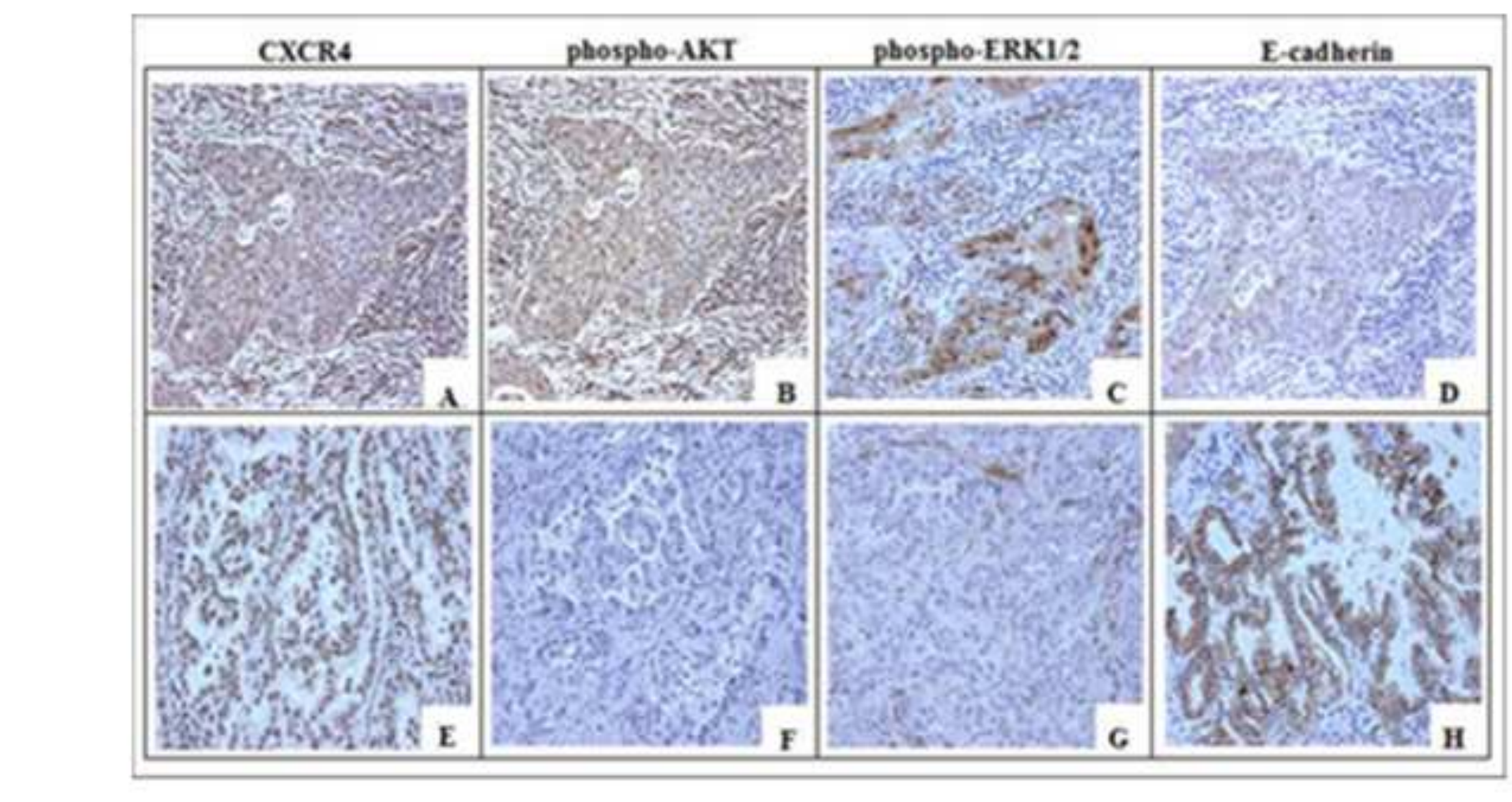



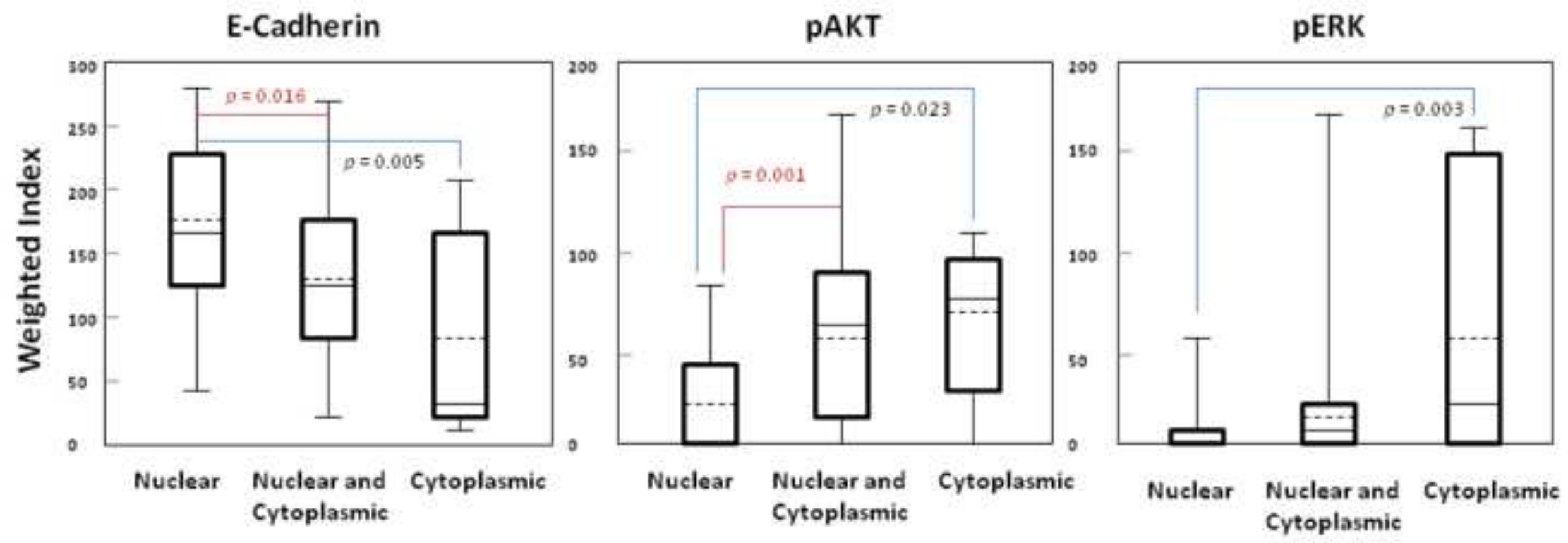

\section{CXCR4 Localization}


Figure 3a

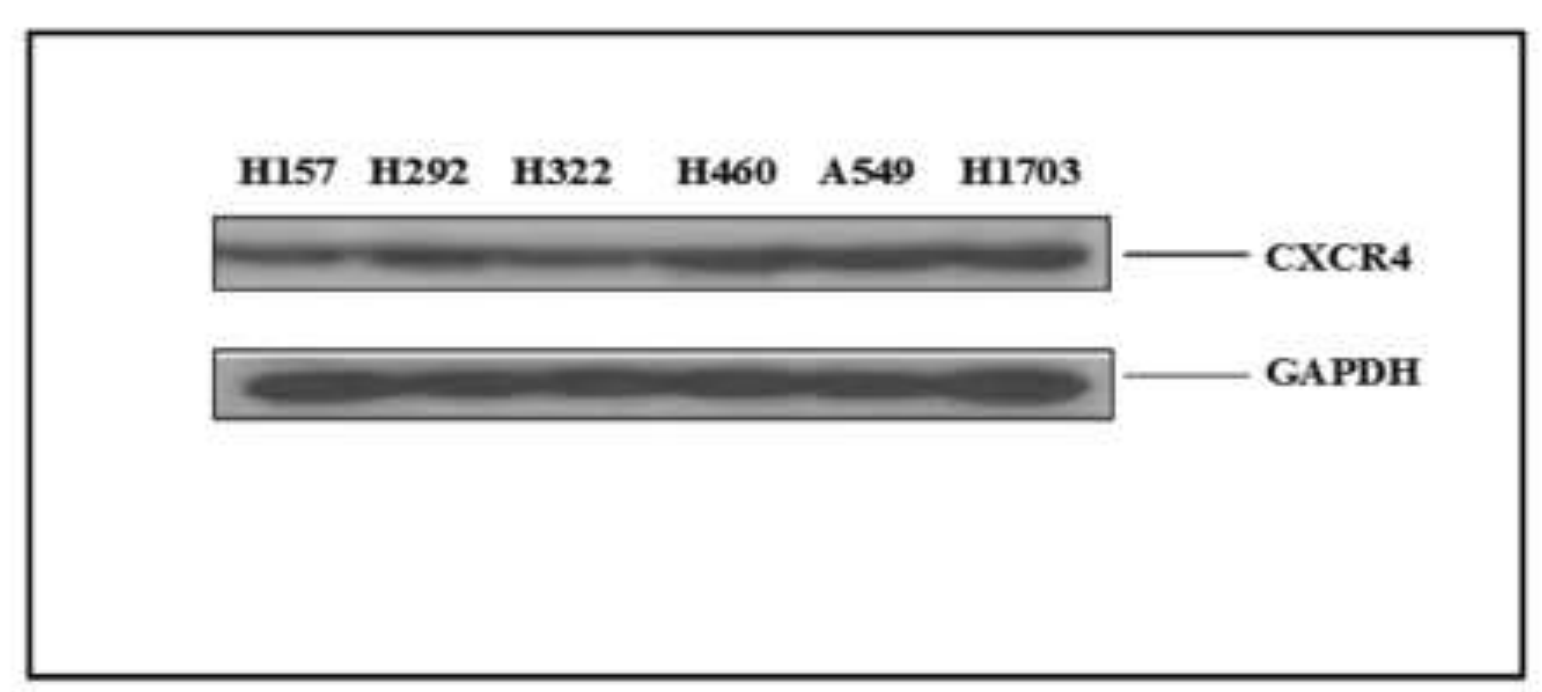

$\begin{array}{lllll}\text { H157 H292 } & \text { H322 } & \text { H460 A549 } & \text { H1703 }\end{array}$

$-\mathrm{CXCR}_{4}$

- CAPDH 


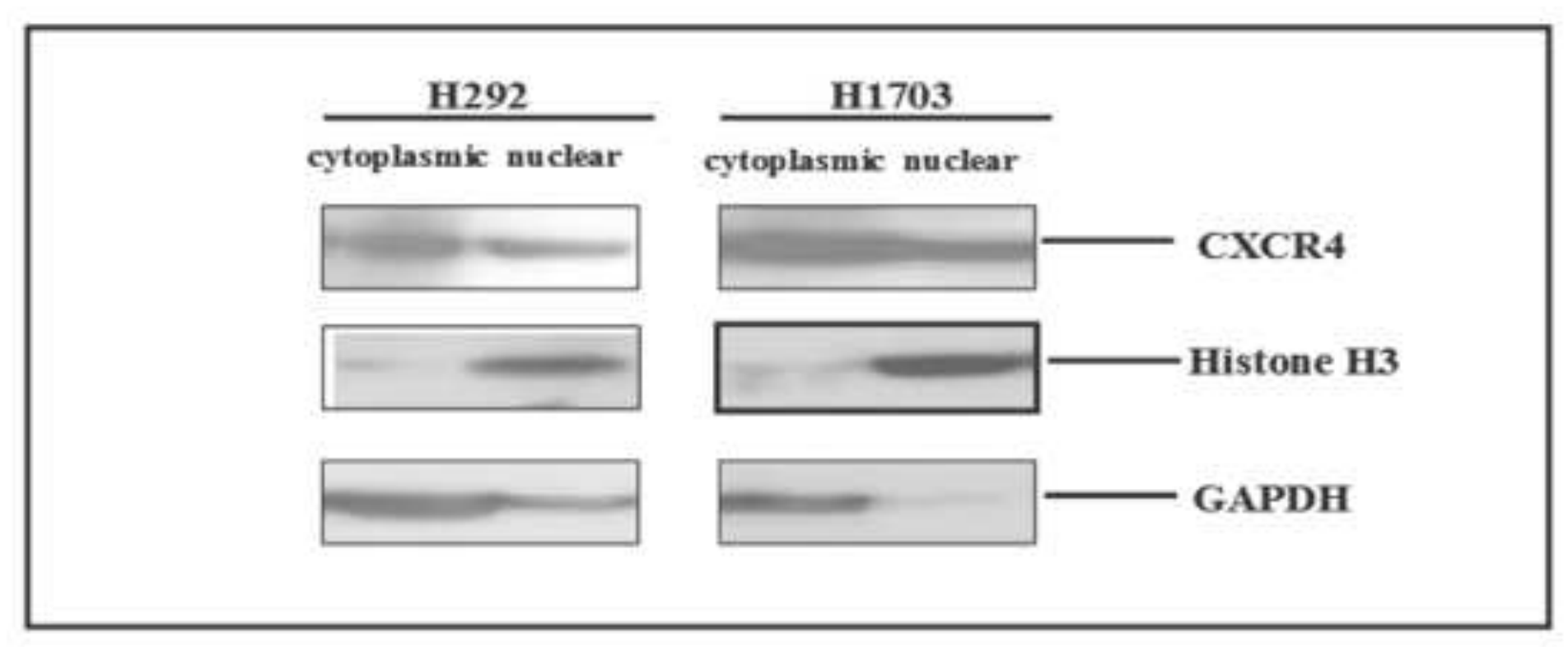

\section{Figure $3 b$}


Figure 4
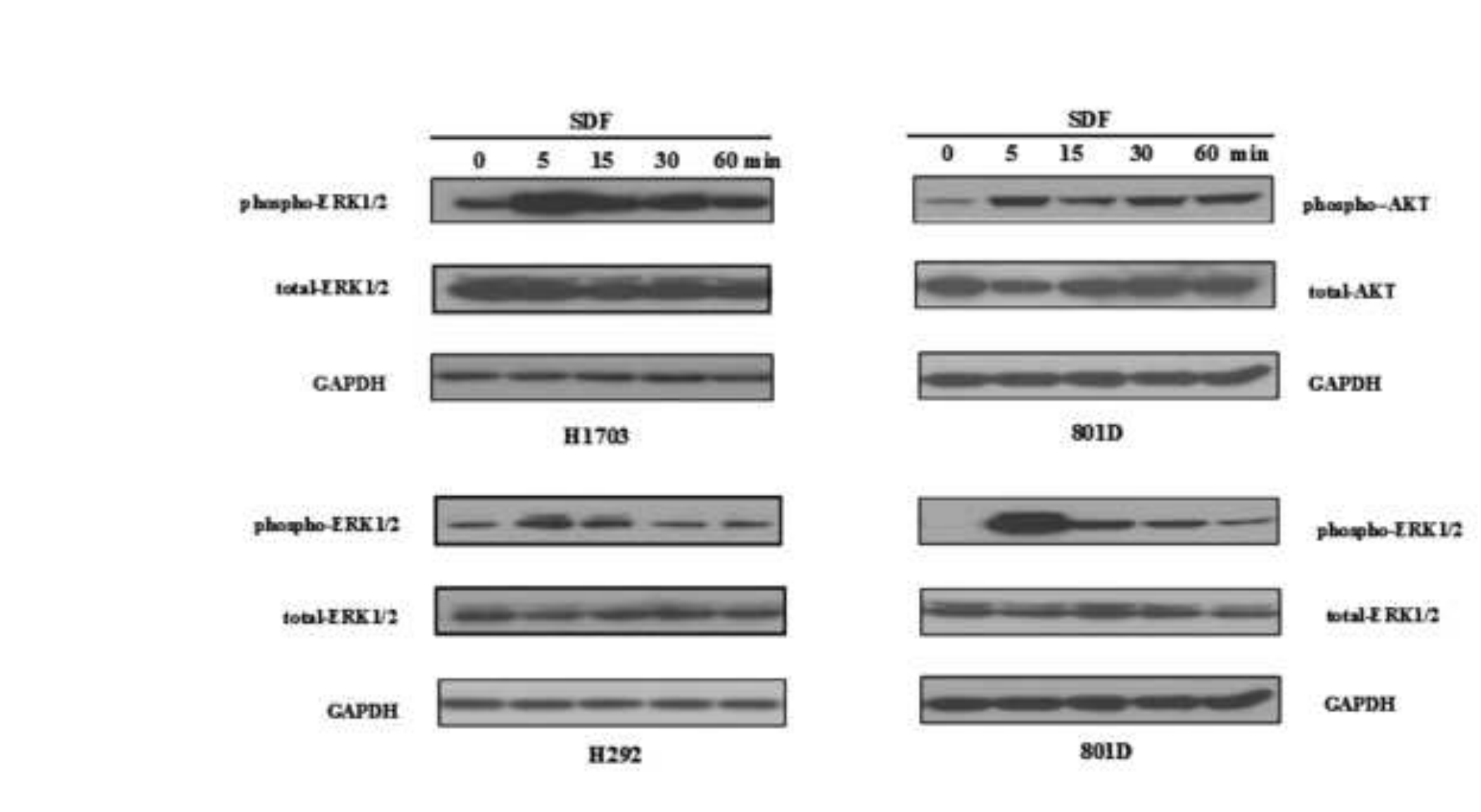

$$
8015
$$

\section{4}

.

.

